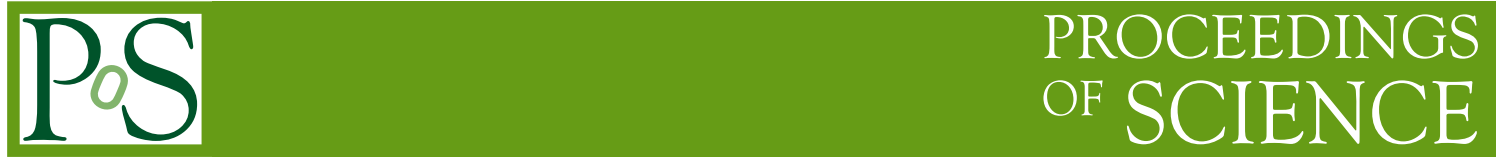

\title{
Measurements of Top Quark Properties at the Tevatron
}

M.H.L.S. Wang* for the D $\varnothing$ and CDF collaborations

University of Rochester, Rochester, New York 14627, USA

E-mail: mwang@fnal.gov

XXth Hadron Collider Physics Symposium

November 16 - 20, 2009

Evian, France

* Speaker. 


\section{Introduction}

The top quark was discovered in 1995 at Fermilab by the DØ and CDF collaborations in Run I of the Tevatron collider. Being the last remaining and most elusive member of the 3 generation family of quarks predicted by the Standard Model (SM) of particle interactions, its discovery was an extraordinary scientific achievement and a major confirmation of the theory. While the discovery was based on a handful of events corresponding to about $100 \mathrm{pb}^{-1}$ of integrated luminosity per experiment, the results presented in this talk are based on substantially more data corresponding to as much as $4.3 \mathrm{fb}^{-1}$ of integrated luminosity from Run II of the Tevatron. The significant increase in statistics in Run II allows more precise determinations of previously measured properties (e.g. mass) and first-time determinations of other properties never before measured.

In this talk, we present the latest measurements of various properties of the top quark from the $\mathrm{D} \varnothing$ and CDF collaborations. The measured properties are grouped into three categories. The first category involves the intrinsic and fundamental properties of mass, top quark $(t)$ and antitop quark $(\bar{t})$ mass difference, width, and electric charge. The second category involves properties related to the production of top-quark pairs $(t \bar{t})$ which includes spin correlations and the differential cross section in $t \bar{t}$ invariant mass. The last category involves properties related to top quark decay which includes $W$ helicity, ratio of decay branching fractions, and top quark decays to charged Higgs bosons.

Despite the larger data sets currently available, the measurement of top quark properties remains a challenging task. This is due to ambiguities in the physics process contributing to the observed event, in associating jets with partons, and due to additional jets from final state radiation. Fortunately, sophisticated techniques have been developed to address such ambiguities making precise measurements of top quark properties possible. Two of these techniques, widely used in the top quark measurements presented here, are the template based method and the matrix element method (ME) which are discussed in more detail elsewhere [2].

\section{Intrinsic Properties}

\subsection{Mass}

The single most precisely measured property of the top quark is its mass. The top quark is the most massive of all quarks and leptons implying a strong coupling to the Higgs boson and suggesting a special role in electroweak symmetry breaking. The mass of the top quark $\left(m_{\mathrm{top}}\right)$ is also related to that of the Higgs boson through radiative corrections to the $W$ boson mass. A precise measurement of $m_{\text {top }}$ can therefore constrain the Higgs boson mass, providing a crucial guide to the search for the Higgs boson.

The two most precise measurements of $m_{\text {top }}$ from D $\varnothing$ and CDF are both in the $\ell+$ jets channel and based on the ME technique [3]. Both measurements implement an in-situ jet energy scale calibration by constraining the invariant mass of the two jets from the hadronic $W$ boson to its well known value of $80.4 \mathrm{GeV}$. The $\mathrm{D} \emptyset$ result is extracted from likelihoods constructed from signal and background probabilities calculated for every single event. The CDF result is based on likelihoods calculated under the assumption that each event is a signal event and background events are rejected with a neural-network based discriminant. DØ measures $m_{\text {top }}=173.7 \pm 0.8$ (stat) \pm 1.6 (syst) Gev 
based on $3.6 \mathrm{fb}^{-1}$ of data and CDF measures $m_{\text {top }}=172.6 \pm 0.9$ (stat) \pm 1.3 (syst) GeV based on $4.3 \mathrm{fb}^{-1}$ of data. The world average value of $m_{\text {top }}=173.1 \pm 0.6$ (stat) \pm 1.1 (syst) GeV from the 2009 winter conferences includes, among other results, the DØ result and an earlier version of the CDF result.

\subsection{Mass Difference}

Since the $C P T$ theorem demands the $t$ and $\bar{t}$ quarks masses to be identical, one can measure the mass difference between $t$ and $\bar{t}$ quarks in order to test $C P T$ invariance. Many precise mass difference measurements have been performed between particles and their antiparticles but all such measurements have been performed on composite objects. To date, no direct measurement of the mass difference between a quark and its antiquark partner has ever been attempted for the simple reason that quarks are never observed in isolation. The top quark, however, is unique because it decays before hadronization making such a measurement possible.

DØ has performed the first and only direct measurement of the mass difference between a quark and its antiquark partner using $t \bar{t}$ events in the $\ell+$ jets channel [4]. This measurement is based on $1 \mathrm{fb}^{-1}$ of data and uses the same ME technique used in the mass analysis described above. Two-dimensional likelihoods calculated as a function of $t$ and $\bar{t}$ quark mass are projected along diagonal lines of constant mass difference. The resulting one-dimensional likelihoods, which are only a function of mass difference, are then used to extract the measurement. DØ measures a mass difference of $3.8 \pm 3.7 \mathrm{GeV}$ which is consistent with $C P T$ invariance.

\subsection{Electric Charge}

The top quark is the weak isospin partner of the $b$-quark in the SM and is expected to have an electric charge of $+2 e / 3$. However, due to the ambiguity in pairing $b$ jets to $W$ bosons, the decay $t \rightarrow b W^{-}$is conceivable and would require the electric charge of the top quark to be $-4 e / 3$. This can be accomodated by scenarios with 4th generation quarks and leptons where the observed top quark is non-SM and the yet unobserved SM top quark has a mass of $\sim 270 \mathrm{GeV}$. The top quark's electric charge is a fundamental property that is an important quantity to measure. A direct measurement can be used to test compatibility of observed events to SM or non-SM scenarios.

The first direct measurement of the top quark's electric charge comes from DØ based on events in the $\ell+$ jets channel from $0.37 \mathrm{fb}^{-1}$ of data [5]. This analysis measures the absolute value of the top quark charge defined as $Q_{1}=\left|q_{\ell}+q_{b_{\ell}}\right|$ and $Q_{2}=\left|q_{\ell}+q_{b_{h}}\right|$ where $q_{\ell}$ is the lepton charge and $q_{b_{l}}$ and $q_{b_{h}}$ are the charges of $b$-tagged jets on the leptonic and hadronic branches of the event. An estimator for the jet charge $q_{\text {jet }}$ is used which is defined based on Monte Carlo (MC) simulations. Expected $q_{\text {jet }}$ distributions for $b$ and $\bar{b}$ (also $c$ and $\bar{c}$ ) quarks are derived from heavy flavor enhanced dijet samples. These $q_{\text {jet }}$ distributions are essentially probability distributions that form the basis for generating the expected top quark charge distributions which, in turn, serve as templates that are compared with data in order to calculate likelihoods used to test compatibility with various scenarios. DØ finds that the observed top quark charge is consistent with the SM excluding a scenario consisting only of non-SM top quarks at up to $92.2 \%$ C.L. In a scenario allowing an admixture of SM and non-SM top quarks, they obtain limits for the non-SM fraction of $0 \leq \rho<$

0.80 at the $90 \%$ C.L. CDF has also performed a similar analysis on $1.5 \mathrm{fb}^{-1}$ data using both the 
$\ell+$ jets and dilepton channels [6]. The results strongly favor a SM top quark excluding the non-SM hypothesis with $87 \%$ C.L. They set a lower limit of $(1-\rho)>0.4$ at $95 \%$ C.L. for the fraction of SM top quark in a scenario allowing a combination of SM and non-SM top quarks.

\subsection{Width}

Being the heaviest, the top quark has the largest decay width and hence shortest lifetime of all SM quarks. The total width of the SM top quark $\left(\Gamma_{t}\right)$ is known to $\sim 1 \%$ at $\sim 1.5 \mathrm{GeV}$ for $m_{\text {top }}=175$ $\mathrm{GeV}$. Deviations from this value can signal contributions from decays to non-SM particles. $\Gamma_{t}$ also provides an indirect way to rule out non-SM decays with non-detectable final states.

The first and only direct measurement of the top quark width is from CDF [7]. It is based on $\ell+$ jets events from $0.955 \mathrm{fb}^{-1}$ of data. The analysis uses the template based method where reconstructed $m_{\text {top }}$ distributions are obtained from MC events generated with different values of $\Gamma_{t}$. These distributions form templates which are then used to fit the data distributions in order to extract $\Gamma_{t}$. Using this method, CDF obtains the first experimental bound on the top quark width of $\Gamma_{t}<13.1 \mathrm{GeV}$ at $95 \%$ C.L. assuming $m_{\mathrm{top}}=175 \mathrm{GeV}$. CDF has also made a direct measurement of the lifetime based on impact parameter distributions and finds a result consistent with zero [8].

\section{Properties Related to Production}

\subsection{Spin Correlations}

The major mechanisms at hadron colliders for $t \bar{t}$ production are quark-antiquark $(q \bar{q})$ annihilation and gluon-gluon ( $g g$ ) fusion. At threshold, the two incoming spin 1/2 quarks annihilate to produce a $t \bar{t}$ pair in a spin $1\left({ }^{3} S_{1}\right)$ state while the two incoming spin 1 gluons in the second process combine to produce a $t \bar{t}$ pair in the spin $0\left({ }^{1} S_{0}\right)$ state. This means that, at threshold, the spins of the $t$ and $\bar{t}$ quarks are expected to be correlated - aligned in the $q \bar{q}$ annihilation process and anti-aligned in the $g g$ fusion process. At the Tevatron, the $q \bar{q}$ annihilation process is dominant, and although orbital angular momentum effects come into play due to energies above threshold, a significant amount of correlation is expected between the $t$ and $\bar{t}$ quark spins. Because of its short lifetime, the original polarization of the top quark at production is preserved when it decays just before strong interaction effects come into play. Because it decays via the weak interaction, this polarization information is transferred to its decay products. Therefore, it is possible to measure the amount of spin correlation between the $t \bar{t}$ pair by looking at its decay products.

Both CDF and D $\varnothing$ have measured $t \bar{t}$ spin correlations using events in the dilepton channel [9]. Both analyses are performed by selecting a quantization axis in the $t \bar{t}$ rest frame and measuring the angle $\theta_{1}\left(\theta_{2}\right)$ of the positive (negative) lepton's momentum vector in the $t(\bar{t})$ quark rest frame with respect to this quantization axis. The differential cross section in terms of these angles is given by $(1 / \sigma) d^{2} \sigma /\left(d \cos \theta_{1} d \cos \theta_{2}\right)=\left(1-C \cos \theta_{1} \cos \theta_{2}\right) / 4$ where $C$ is the correlation coefficient to be determined. CDF uses the off-diagonal basis while DØ uses the beam basis. At NLO, the SM predicts $C=0.782$ and $C=0.777$, respectively, in the off-diagonal and beam bases. The CDF analysis is based on $2.8 \mathrm{fb}^{-1}$ of data and uses the template based method. In addition to the leptons from the $W$ boson decays, it also uses the angles of the momentum vector of the $b(\bar{b})$ quark in the $t(\bar{t})$ rest frame with respect to the quantization axis. Templates are based on two-dimensional distributions 
in the cosines of the lepton and $b$ quark angles. They measure $C=0.32_{-0.775}^{+0.545}$ (stat+syst) which is consistent with the SM. The $\mathrm{D} \varnothing$ analysis is based on $4.2 \mathrm{fb}^{-1}$ of data and also uses the template based method. Templates are generated in $\cos \theta_{1} \times \cos \theta_{2}$ from MC samples with different amounts of correlation. The value extracted from data is $C=-0.17_{-0.53}^{+0.64}$ (stat+syst) which agrees with the SM to within 2 standard deviations.

\subsection{Differential Cross Section in $t \bar{t}$ Invariant Mass}

The shape of the $t \bar{t}$ invariant mass $\left(M_{t \bar{t}}\right)$ spectrum is a unique feature of the SM top quark. New particles and mechanisms predicted in various beyond the standard model theories can distort the $M_{t \bar{t}}$ spectrum. While traditional analyses have focused on direct searches for resonances in the $M_{t \bar{t}}$ spectrum, this spectrum can also be tested in a more general way for consistency with the SM. Such an approach is sensitive to both narrow resonances and broad enhancements.

$\mathrm{CDF}$ has taken this approach using $\ell+$ jets events in $2.7 \mathrm{fb}^{-1}$ of data [10]. Based on reconstructed quantities, they calculate $M_{t \bar{t}}$ from $0-1400 \mathrm{GeV}$. After subtracting a background modeled from $\mathrm{MC}$, the spectrum is unfolded and the differential cross section $d \sigma / d M_{t \bar{t}}$ calculated. Like the mass analyses described at the beginning, an in-situ jet energy calibration is used to constrain the jet energy scale and thereby reduce the systematic uncertainty due to this source. They compare $d \sigma / d M_{t \bar{t}}$ with SM expectations and conclude no evidence for non-SM physics.

\section{Properties Related to Decay}

\section{1 $W$ Helicity}

The SM $t \rightarrow W^{+} b$ decay proceeds via the $V-A$ charged current weak interaction. A massless $b$ quark will have negative helicity in the top quark rest frame. In order to conserve angular momentum, the polarization of the $W$ boson will either be longitudinal (vanishing spin projection) or left-handed (negative helicity). The SM predicts a longitudinally polarized fraction of 0.697 and a right-hand polarized (positive helicity) fraction of $O\left(10^{-4}\right)$ for $m_{\text {top }}=172.5$ $\mathrm{GeV}$. One can therefore search for deviations from the SM by measuring the $W$ boson helicity fractions. $\mathrm{D} \varnothing$ and $\mathrm{CDF}$ have measured these fractions from the angular distribution given by $\omega\left(\theta^{*}\right)=3 f_{0}\left(1-\cos ^{2} \theta^{*}\right) / 4+3 f_{-}\left(1-\cos \theta^{*}\right)^{2} / 8+3 f_{+}\left(1+\cos \theta^{*}\right)^{2} / 8$ where $f_{0}, f_{-}$, and $f_{+}$are the longitudinal, left-handed, and right-handed $W$ helicity fractions, respectively [11]. D $\emptyset$ defines $\theta^{*}$ as the angle between the momentum vectors of the $W$ boson and its decay lepton in the top quark rest frame. CDF defines $\theta^{*}$ as the angle between the momentum vectors of the lepton and the top quark in the $W$ boson rest frame.

DØ uses a template based method on $\ell+$ jets and dilepton events from $2.7 \mathrm{fb}^{-1}$ of data. Templates for $\cos \theta^{*}$ are used to fit the data to extract the helicity fractions. Assuming $f_{0}+f_{+}+f_{-}=1$, $f_{0}$ and $f_{+}$are determined simultaneously to be $f_{0}=0.490 \pm 0.106$ (stat) \pm 0.085 (syst) and $f_{+}=$ $0.110 \pm 0.059$ (stat) \pm 0.052 (syst) which are consistent with the SM at the $23 \%$ C.L.

$\mathrm{CDF}$ uses the ME based technique on $\ell+$ jets events from $2.7 \mathrm{fb}^{-1}$ of data. Event probabilities are calculated as function of $f_{0}$ and $f_{+}$simultaneously. CDF determines $f_{0}=0.88 \pm 0.11$ (stat) \pm 0.06 (syst) and $f_{+}=-0.15 \pm 0.07$ (stat) \pm 0.06 (syst). Each fraction is also determined by constraining the other to the $\mathrm{SM}$ value and are found to be $f_{0}\left(f_{+}=0\right)=0.70 \pm 0.07$ (stat) \pm 0.04 (syst) and $f_{+}\left(f_{0}=0.7\right)=-0.01 \pm 0.02$ (stat) \pm 0.05 (syst) which are consistent with SM predictions. 


\subsection{Ratio of Branching Fractions}

The SM top quark decays into a $W$ boson and a down type quark. The ratio of top quark decays into $W b$ to those into $W q(q=d, s, b)$ can be written in terms of the CKM matrix elements as $R=B(t \rightarrow W b) / B(t \rightarrow W q)=\left|V_{t q}\right|^{2} /\left(\left|V_{t b}\right|^{2}+\left|V_{t s}\right|^{2}+\left|V_{t d}\right|^{2}\right)$. The $\left|V_{t q}\right|$ 's are tightly constrained with $\left|V_{t q}\right| \sim 1$ based on the assumption of a unitary three generation CKM matrix and experimental measurements of CKM matrix elements. The SM values of $\left|V_{t q}\right|$ can be altered by non-SM processes in top quark production and decays or a 4th generation of quarks resulting in $R$ deviating from the expected value close to unity. The experimental determination of $R$ can therefore be used to check SM assumptions and test for new physics.

CDF has measured $R$ using both $\ell+$ jets and dilepton events in $0.16 \mathrm{fb}^{-1}$ of data [13]. The data samples are subdivided based on the number of identified $b$ jets in the event. An artificial neural network is employed for the 0 -tag $\ell+$ jets sample for the prediction of background levels. The number of expected events in each subsample is then compared with the observed number in order to construct likelihoods from which $R=1.12_{-0.19}^{+0.21}$ (stat $)_{-0.13}^{+0.17}$ (syst) is extracted. Lower limits of $R>0.61$ and $\left|V_{t b}\right|>0.78$ at $95 \%$ C.L. are also obtained.

DØ has measured $R$ using $\ell+$ jets in $0.9 \mathrm{fb}^{-1}$ of data [12]. This is essentially a cross section measurement with a simultaneous determination of $R$. The data sample is split into subsamples based on lepton flavor, jet multiplicity, and number of identified $b$ jets. A discriminant is also used in the 0 -tag, $\geq 4$ jets sample for improved signal to background discrimination. The number and distribution of events in the subsamples depends on $\sigma$ and $R$ which are fitted for by comparing observed to expected numbers in the subsamples as a function of $\sigma$ and $R$. DØ finds $R=0.97_{-0.08}^{+0.09}$ (stat+syst) and sets lower limits of $R>0.79$ and $\left|V_{t b}\right|>0.89$ at $95 \%$ C.L.

\subsection{Charged Higgs}

The simplest extensions to the SM require the existence of two different Higgs fields that manifest themselves as two charged Higgs bosons $\left(H^{ \pm}\right)$and three netural ones. If the mass of the charged Higgs is low enough, the top quark is expected to decay via $t \rightarrow H^{+} b$. The branching ratios of the charged Higgs themselves depend on the ratio $\tan \beta$ of the vacuum expectation values of the two Higgs fields. Decays into quarks $\left(H^{+} \rightarrow c \bar{s}\right)$ dominate at low $\tan \beta$ while decays into a tau and a neutrino $\left(H^{+} \rightarrow \tau \nu\right)$ dominate at high $\tan \beta$. Because of the different decay branching ratios of the $H^{+}$, its existence can modify the distribution of observed events in the different top decay channels. This implies that, aside from direct searches, indirect $H^{+}$seaches are also possible by comparing the observed distribution of events relative to SM expectations.

DØ has conducted an indirect search for the $\mathrm{H}^{+}$in the $\ell+$ jets, dilepton, and $\tau+$ lepton channels using $1 \mathrm{fb}^{-1}$ of data [14]. In this analysis, the data is split into sub-samples based on final state. Like a typical cross section measurement, the predicted number of events in each sub-sample are then compared with the observed number through a likelihood fit. Since no evidence is found for the $H^{+}$, upper limits are extracted for the leptophobic $\left(B\left(H^{+} \rightarrow c \bar{s}\right)=1\right)$, tauonic $\left(B\left(H^{+} \rightarrow\right.\right.$ $\tau \nu)=1)$, and mixed $\left(B\left(H^{+} \rightarrow \tau v\right)+B\left(H^{+} \rightarrow c \bar{s}\right)=1\right)$ cases. At 95\% C.L., $B\left(t \rightarrow H^{+} b\right)>0.22$ is excluded for $80<M_{H^{+}}<155 \mathrm{GeV}$ in the leptophobic case and $B\left(t \rightarrow H^{+} b\right)>0.15-0.19$, depending on $M_{H^{+}}$, is excluded in the tauonic case. For the tauonic case, a "model-independent" 
simultaneous fit of $\sigma(t \bar{t})$ and $B\left(t \rightarrow H^{+} b\right)$ is also performed, resulting in the exclusion (95\% C.L.) of $B\left(t \rightarrow H^{+} b\right)>0.12-0.26$ depending on $M_{H^{+}}$.

CDF has performed the first direct search of $H^{+} \rightarrow c \bar{s}$ in the $\ell+$ jets channel with $2.2 \mathrm{fb}^{-1}$ of data [15]. $t \bar{t}$ events are reconstructed using a kinematic fitter without imposing a $W$ boson mass constraint on the dijet invariant mass in the hadronic branch. Additional jets, assumed to originate from final state radiation, are merged with the closest ones to improve jet resolution. The reconstructed dijet mass in data is then compared with templates generated according to $W, H^{+}$, or background hypotheses. Since no evidence for the $H^{+}$is found, the exclusion region $B(t \rightarrow$ $\left.H^{+} b\right)>0.1-0.3$ for $60<M_{H^{+}}<150 \mathrm{GeV}$ is obtained at $95 \%$ C.L. assuming $B\left(H^{+} \rightarrow c \bar{s}\right)=1$.

\section{Conclusion}

We have presented the latest measurements from the Tevatron of various properties of the top quark - both fundamental and those related to production and decay. So far, the observed

top quark seems consistent with SM predictions. With the increasing data in the coming year, these measurements will continue improving, allowing the top quark and the SM to be tested more stringently.

\section{References}

[1] F. Abe et al. (CDF Collaboration), Phys. Rev. Lett. 74, 2626 (1995); S. Abachi et al. (D0 Collaboration), Phys. Rev. Lett. 74, 2632 (1995).

[2] M.H.L.S. Wang for the D0 and CDF Collaborations, arXiv:hep-ex/0904.0411 (2009)

[3] The D0 Collaboration, DØ Note 5877-CONF (March 2009); The CDF Collaboration, CDF Note 9880 (August 2009).

[4] V.M. Abazov et al. (D0 Collaboration), Phys. Rev. Lett. 103, 132001 (2009).

[5] V.M. Abazov et al. (D0 Collaboration), Phys. Rev. Lett. 98, 041801 (2007).

[6] The CDF Collaboration, CDF Note 8967 (August 2007)

[7] T. Aaltonen et al. (CDF Collaboration), Phys. Rev. Lett. 102, 042001 (2009).

[8] The CDF Collaboration, CDF Note 8104 (February 2006).

[9] The D0 Collaboration, DØ Note 5950-CONF (July 2009); The CDF Collaboration, CDF Note 9824 (June 2009).

[10] T. Aaltonen et al. (CDF Collaboration), Phys. Rev. Lett. 102, 222003 (2009).

[11] The D0 Collaboration, DØ Note 5722-CONF (July 2008); The CDF Collaboration, CDF 10004 (November 2009).

[12] V.M. Abazov et al. (D0 Collaboration), Phys. Rev. Lett. 100, 192003 (2008).

[13] D. Acosta et al. (CDF Collaboration), Phys. Rev. Lett. 95, 102002 (2005).

[14] V.M. Abazov et al. (D0 Collaboration), Phys. Lett. B682, 278 (2009).

[15] T. Aaltonen et al. (CDF Collaboration), Phys. Rev. Lett. 103, 101803 (2009). 